REDES- Revista hispana para el análisis de redes sociales

Vol.20,\#5, Junio 2011

http://revista-redes.rediris.es

\title{
La dispersión geográfica de las redes personales: cálculo y significado*
}

\author{
José Luis Molina (egolab-GRAFO)*, Mireia Bolíbar e Irene Cruz (QUIT) ${ }^{ \pm}$- \\ Universitat Autònoma de Barcelona
}

\begin{abstract}
Resumen
En este artículo se comparan tres índices que representan la dispersión geográfica de las redes personales. El primer índice se construye en base al cómputo de las distancias para cada Ego con sus Alteri y de éstos entre sí. Una vez calculada la distancia total, se obtiene la media para cada Ego. El valor absoluto del logaritmo de esta media es la medida propuesta. El segundo índice se construye teniendo en cuenta solamente las relaciones Ego-Alter de la fórmula anterior y el tercero, habitual en este tipo de estudios, muestra la distribución de los contactos activos en un ranking de distancias (mismo barrio, misma ciudad, Cataluña, resto de España, extranjero).

La comparación se realiza explorando el efecto de los tres índices en el apoyo social intercambiado de la población residente en Cataluña. Los datos se han tomado de la encuesta de redes personales realizada en el marco del proyecto CASREDIN interesado en explorar las redes sociales y la cohesión social en Cataluña. Así, dividimos la población residente entre "nacionales" e "inmigrantes" y en cada uno de los grupos evaluamos el apoyo social en función de la dispersión geográfica de las redes personales. Finalmente, proponemos una discusión de los resultados en el marco de la teoría del "transnacionalismo".
\end{abstract}

Palabras clave: redes personales, trasnacionalismo, emigración, dispersión geográfica.

\begin{abstract}
This article compares three indices that represent the geographical dispersion of personal networks. The first index is built on the computation of distances for each Ego with their Alters and among each one them. After calculating the total distance, it is obtained the average for each Ego. The absolute value of the logarithm of this average is the proposed measure. The second index is constructed taking into account only the relations Ego-Alter of the above formula and the third, common in such studies, shows the distribution of active contacts in a ranking of distances (same neighbourhood, same city, Catalonia, rest of Spain, other countries).
\end{abstract}

The comparison is made exploring the effect of the three indexes on the exchanged social support of people living in Catalonia. The data are taken from the survey of

\footnotetext{
* Este artículo forma parte del proyecto CSO2008-01470 del Ministerio de Educación y Ciencia. Los autores de este artículo forman parte de dicho proyecto. Agradecemos los comentarios recibidos por los participantes en el seminario "Inmigración, Redes y Cohesión Social" celebrado los días 16 y 17 de Diciembre en la Universidad Autónoma de Barcelona, especialmente las sugerencias de Carlos Lozares, Michel Grossetti, Joan Miquel Verd, Sònia Parella y Ainhoa de Federico.

* Departament d'Antropologia social i cultural. Enviar correspondencia a joseluis.molina@uab.es.

${ }^{ \pm}$Centre d'Estudis Sociològics sobre la Vida Quotidiana i el Treball (QUIT). Departament de Sociologia Mireia.Bolibar@uab.cat, irene.cruz@uab.cat.
} 
personal networks in the project CASREDIN, interested in exploring social networks and social cohesion in Catalonia. Thus, the resident population divided between "national" and "migrants" and in each of the groups social support is evaluated depending on the geographic dispersion of personal networks. Finally, we propose a discussion of the results within the framework of the theory of "transnationalism".

Key words: personal networks, transnationalism, migration, geographical dispersion.

\section{Geografía y redes sociales: un campo en expansión}

La convergencia de las redes sociales y las tecnológicas experimentada en la última década (Kleinberg 2008) unida a la disponibilidad en tiempo real de la posición geográfica de personas y dispositivos de comunicación, están permitiendo incorporar de forma ordinaria información geográfica a la investigación en una amplia variedad de ámbitos. Así, encontramos por ejemplo contribuciones que relacionan interacciones sociales y localización geográfica en el campo de la Geografía política (Baybeck y Huckfeldt 2002), la Geografía económica (Glückler, 2007), de la Sociología (Hampton y Wellman 2002, Grossetti 2006, Mok y Wellman 2007), de la Epidemiología (Obbo, 1993, Altha et al. 2001, Chen et al., 2007), de los estudios de transporte y movilidad (Larsen et al., 2006, Carrasco et al., 2008; Frändberg, 2008; Urry 2010), las comunicaciones telefónicas (Onnela et al., 2007; Lambiotte et al., 2008; Licoppe et al., 2008) y las simulaciones de un Mundo Pequeño (Liben-Nowell et al., 2005) entre muchas otras.

Estos estudios han puesto de manifiesto algunas cuestiones de interés. El primero de ellos es el fenómeno llamado por Hampton y Wellman (2002) "glocalización", es decir, la concentración de los contactos activos en un corto radio geográfico pero conviviendo simultáneamente con un porcentaje más pequeño de contactos activos distribuidos globalmente. La distancia afecta también a la frecuencia de las interacciones, especialmente a los encuentros cara a cara, pero también a las llamadas telefónicas y en mucha menor medida a los correos electrónicos, más independientes de la distancia (Mok y Wellman 2007), posiblemente por razones de tarificación. El apoyo social, incluso el proporcionado por los familiares, las relaciones más independientes de la geografía, decrece con la distancia (Mulder et al. 2009). Contemplar la distancia de los contactos activos en el análisis ayuda a entender aspectos biográficos (como los desplazamientos por estudios durante largos períodos de tiempo, Frändberg 2008), la recomposición de las relaciones sociales en movimientos migratorios (Grossetti 2006 para migraciones internas), las decisiones que acompañan a la decisión de tener hijos (Clark et al. 2009) o los realojamientos residenciales (Zorlu, 2009). Parece pues, que la dimensión geográfica de las redes sociales merece ser estudiada con atención. 
Antes de centrarnos en el caso de la redes personales en particular, cabe señalar que esta convergencia tecnológica y social no solamente se produce en las conocidas plataformas de social networking (SNS, social networks sites) como Twitter o Facebook (las cuales proporcionan estos datos gratuitamente), sino que también hay autores que predicen nuevas aplicaciones basadas en el concepto de Personal Area Network (PAN, Niemegeers y de Groot 2002, 2003) en la que los dispositivos tecnológicos y los recursos de ego y sus Alteri restarán accesibles y compartidos de forma dinámica.

En nuestro caso estamos interesados en añadir información geográfica a las redes personales recogidas en el proyecto CASREDIN porque pensamos que es una forma de (a) explorar la influencia de la distancia y la movilidad en los fenómenos sociales y culturales objeto de estudio (la cohesión social y las migraciones) y, (b) una manera de dotar de base empírica al concepto de "campo trasnacional" o trasnacionalismo (Glick Schiller et al. 2002, Wimmer et al. 2002, Levitt et al. 2004, Vertovec 2004) y ver si efectivamente se producen los fenómenos descritos por la teoría a partir mayoritariamente de estudios de caso hasta el momento.

La visualización de la distribución geográfica de las redes personales (ver por ejemplo Molina et al. 2005 para miembros de la lista REDES) es ya en sí misma un primer avance, pues permite identificar patrones y fuentes de variación entre colectivos y así generar nuevas hipótesis (Brandes et al. 2006). Igualmente, el concepto propuesto por Axhausen (2008) de "geografía de la red personal", es especialmente útil para nuestra investigación pues representa el tipo de datos que hemos recogido de cada uno de nuestros informantes con la ayuda del programa de código abierto EgoNet (http://sourceforge.net/projects/egonet/), es decir, el conjunto de contactos activos de cada uno de los egos con su respectiva localización geográfica. Esta información puede representarse en forma de mapa, ya sea para cada ego por separado o de forma agregada para todos los egos entrevistados. Nuestra propuesta es desarrollar, además, un índice que capture la dispersión geográfica de cada red personal, añadirlo al vector de variables descriptivas de ego y comparar los resultados con otros índices similares o usados en la literatura.

Una vez calculados los índices, exploraremos su influencia en el apoyo social percibido a partir de los datos obtenidos en el proyecto de investigación CASREDIN y veremos si la información geográfica nos permite identificar aspectos o dimensiones no revelados por otros tipos de análisis. Así, el presente artículo está organizado en los siguientes apartados: en primer lugar, describiremos la investigación sobre la que se basa nuestro trabajo y el tipo de datos obtenidos. En 
segundo lugar, describiremos el índice de dispersión geográfica propuesto en este artículo y su implementación en un programa que usa el output de EgoNet para calcular automáticamente el mencionado índice (Molina et al. 2010). En tercer lugar, presentaremos los resultados de aplicar el índice al estudio del apoyo social percibido y valoraremos los resultados comparados con otros dos índices: uno, una versión simplificada del primero y dos, un índice basado en la distribución de los contactos activos en un ranking de distancias. Por último realizaremos una discusión de los resultados y plantearemos las limitaciones y ámbitos de lo que pensamos es una prometedora línea de investigación futura.

\section{La investigación}

Los datos con los que trabajamos proceden del proyecto I+D+I Estudio comparado de casos sobre la influencia mutua entre capital e integración sociales y la inserción, estabilidad, promoción y cualificación en el empleo (CASREDIN) con referencia CSO2008-01470, subvencionado por el Ministerio de Educación y Ciencia dentro del marco del VI Plan Nacional de Investigación Científica, Desarrollo e Innovación Tecnológica 2008-2011, el investigador principal del cual es Carlos Lozares. El artículo firmado por el conjunto del equipo en este mismo volumen (Lozares, López, Verd et al., 2011) expone los detalles del muestreo y la metodología seguidas en el trabajo de campo. Sintéticamente, el muestreo ha sido aleatorio y estratificado. La estratificación se ha hecho en dos etapas: primero por localidades y luego por colectivos de población que responden a un análisis factorial realizado sobre el censo de Cataluña del 2001 (ver López Roldán y Lozares, 2008). Los datos consisten en 450 encuestas de redes personales con 4 bloques de preguntas: un primer bloque de preguntas de caracterización socio-demográfica de los Ego; la pregunta generadora de redes a partir de la cual se mencionan 30 Alteri; un tercer bloque de preguntas de caracterización socio-demográfica de cada Alter mencionado y por último las relaciones existentes entre cada par de Alteri.

\section{El índice de dispersión geográfico de las redes personales}

Con este índice se propone una operacionalización del concepto de "geografía de la red personal" propuesto por Axhausen (2008) el cual incluye la extensión del área geográfica determinada por la presencia de los contactos activos de Ego.

El cálculo del indicador se realiza mediante el programa Geo-Dispersion (Molina et. al. 2010) disponible en la página de EgoNet en sourceforge. 
Geo-Dispersion requiere como input de información el archivo "raw data" (formato .csv) que genera el programa EgoNet a partir de las entrevistas de redes. GeoDispersions, después de identificar las variables que distinguen a los Ego, a los Alteri y el lugar de residencia de unos y otros, se conecta con el servidor Yahoo PlaceFinder (http://developer.yahoo.com/geo/placefinder/) y geocodifica la información. En el caso de encontrar nombres ambiguos o con errores mecanográficos, éste propone un conjunto de posibles localizaciones. Si ninguna de las localizaciones es la adecuada se puede introducir manualmente la nueva localización, por ejemplo "Comarruga, Barcelona". Una vez asignadas la latitud y la longitud para cada ubicación se computan las distancias entre los pares existentes, ego-alter y alter-alter. La suma de todas esas distancias entre pares se divide por el número de Alteri para obtener la media. A continuación el programa calcula el valor absoluto del logaritmo de esa media para disponer de una escala más manejable.

$\mid \log \left(\Sigma^{\mathrm{N}=\mathrm{x}_{\mathrm{j}=1}}\right.$ [distancias egoi-alter $j+\Sigma$ distancias alterj-alterk] / N Alteri-1)

para las relaciones existentes entre cada par.

Finalmente, Geo-Dispersion genera una nueva matriz de datos en formato .csv con las variables Ego Latitude, Ego Longitude, Alter Latitude, Alter Longitude, Ego-Alter Distance, GeoDispersionAlteri y GeoDispersion.

Además del procedimiento descrito, con el que hemos construido el índice de dispersión de la red personal (Ego-Alter + Alter-Alter), hemos construido otro índice que contempla solamente las distancias entre Ego y sus Alteri (Ego-Alter). Por otra parte se ha construido un tercer indicador nominal y categórico que caracteriza a cada Alter en función de si reside en el barrio de Ego, el municipio, en el resto de Cataluña, en el resto de España (sin contar las categorías anteriores) o en el extranjero. A pesar de que estrictamente no podemos hablar de "índice" en este tercer caso, usaremos la denominación genérica de "índices" para referirnos a las tres modalidades de medición de la distancia en las redes personales. Así, decimos que los histogramas que siguen muestran las distribuciones de frecuencias de los distintos índices construidos.

\section{Índice Ego-Alter + Alter-Alter}

La distribución de este índice se muestra en la Figura 1, en la que se puede apreciar una concentración de los valores alrededor de la media. Esto puede ser 
interpretado de la siguiente forma: una minoría no tiene Alteri distribuidos fuera de su ámbito geográfico y se sitúan a la izquierda -podríamos llamarlos "locales", por seguir el célebre artículo de Merton sobre locales y cosmopolitas (1968)-, la mayor parte de los casos se sitúan en el centro -"glocales"- y un porcentaje variable y decreciente de personas a medida que nos desplazamos a la derecha tiene características que podríamos llamar "cosmopolitas" o "transnacionales".

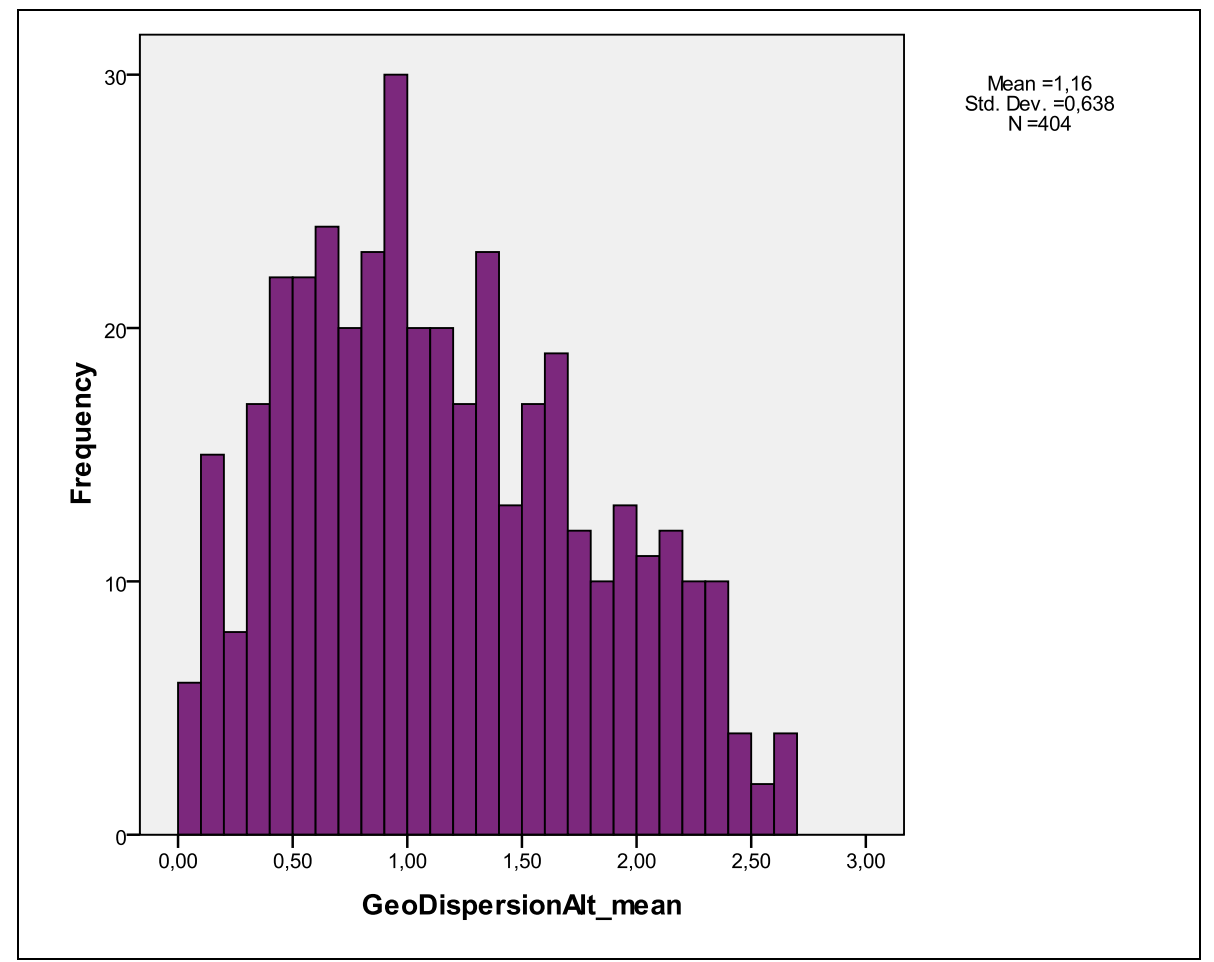

Figura 1. Frecuencia de la distribución de la variable Ego-Alter + Alter-Alter

Esta distribución está afectada por el origen de los Egos que se analizan. En la Figura 2 puede observarse la distribución geográfica de la red personal en función del origen de los Ego ( $1=>55$ años, nacidos en Cataluña; $2=<55$ años nacidos en Cataluña de padres catalanes; $3=<55$ años nacidos en Cataluña con padres nacidos fuera de Cataluña; $4=>55$ años nacidos en España; $5=$ nacidos en Ecuador y $6=$ nacidos en Marruecos). El caso de los colectivos de la derecha (nacidos en Ecuador y nacidos en Marruecos) muestra un índice de dispersión geográfica mayor que el resto. 


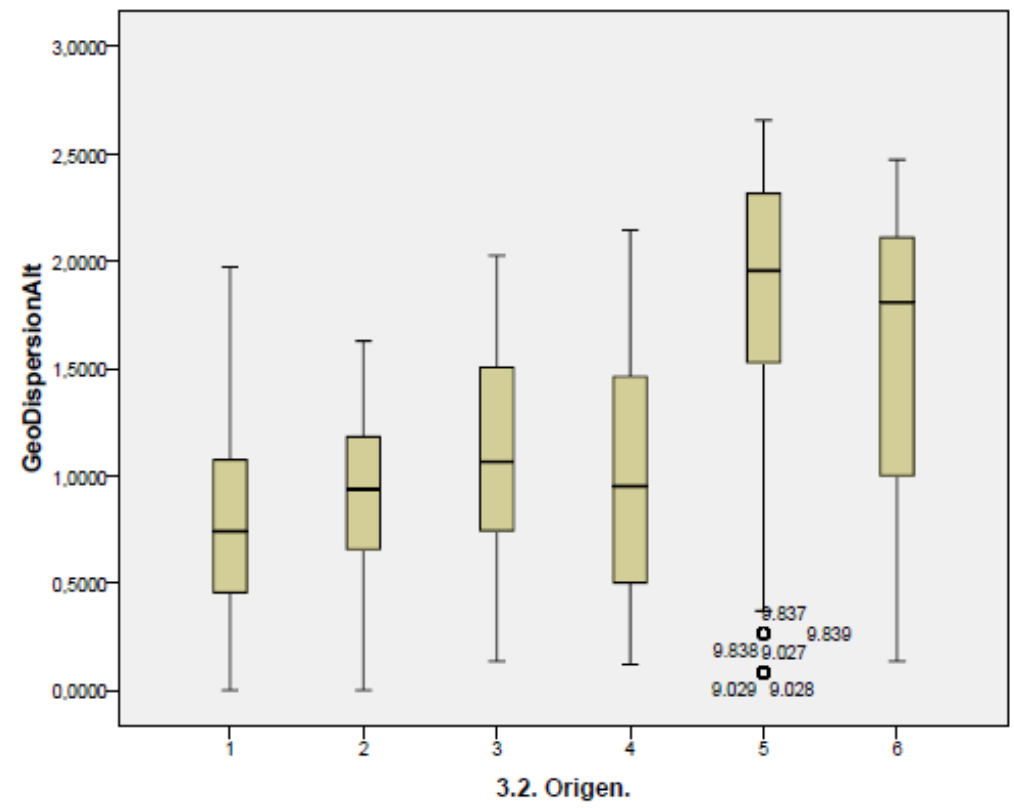

Figura 2. Diferencias en la distribución geográfica de la red personal en función del origen de los Ego según la el índice de dispersión Ego-Alter + Alter-Alter.

\section{Índice Ego-Alter}

El segundo índice es más fácil de computar pues solamente tiene en cuenta las distancias Ego-Alter (fórmula 2). La distribución se muestra en la Figura 3.

$$
\mid \log \left(\Sigma^{N=x}{ }_{j=1}\right. \text { distancias egoi-alterj / N Alteri-1)| }
$$

Este índice representa fielmente el concepto de "glocalización" (mayoría de locales y minoría de "globales", por usar los términos del neologismo), pues de hecho es un concepto que se sitúa en la perspectiva de los Egos para observar la distribución de las distancias de sus Alteri. Dicho de otra forma: este segundo índice operacionaliza este concepto. 


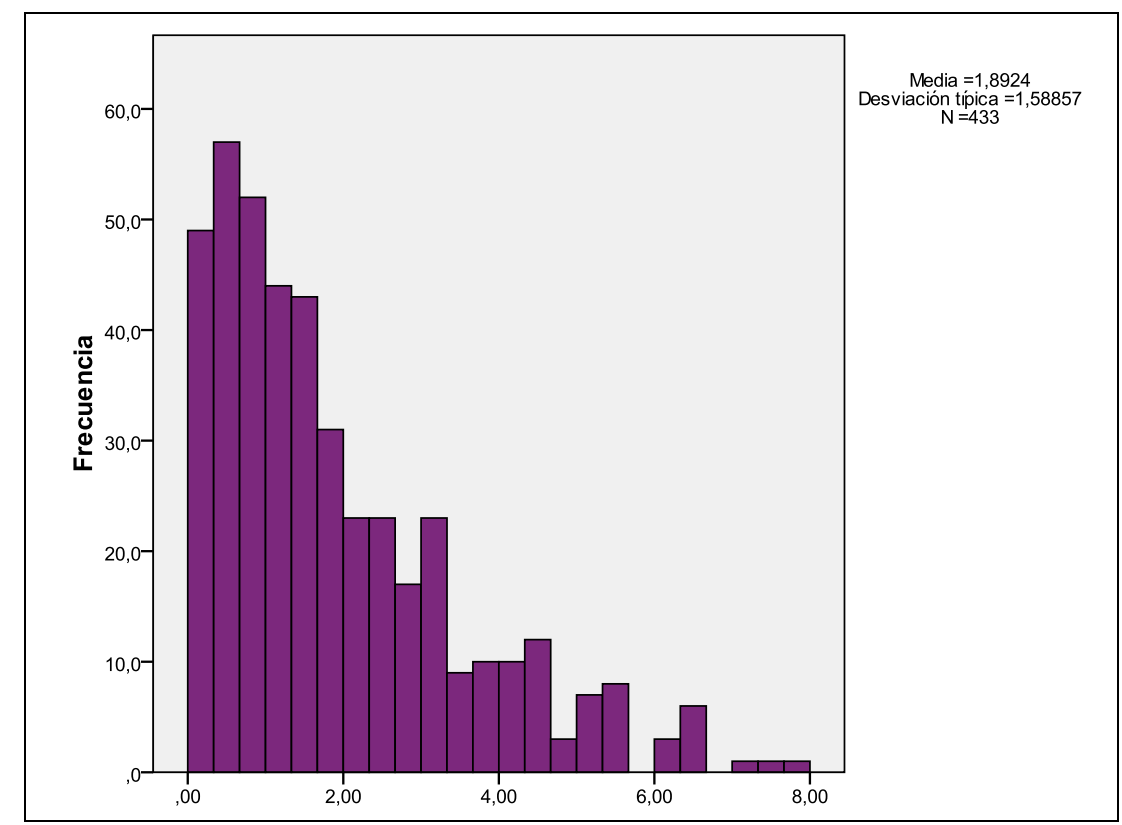

Figura 3. Frecuencia de la distribución de la variable Ego-Alter.

Este índice, al igual que el anterior, muestra los dos grupos de extranjeros con un índice de dispersión geográfica mayor.

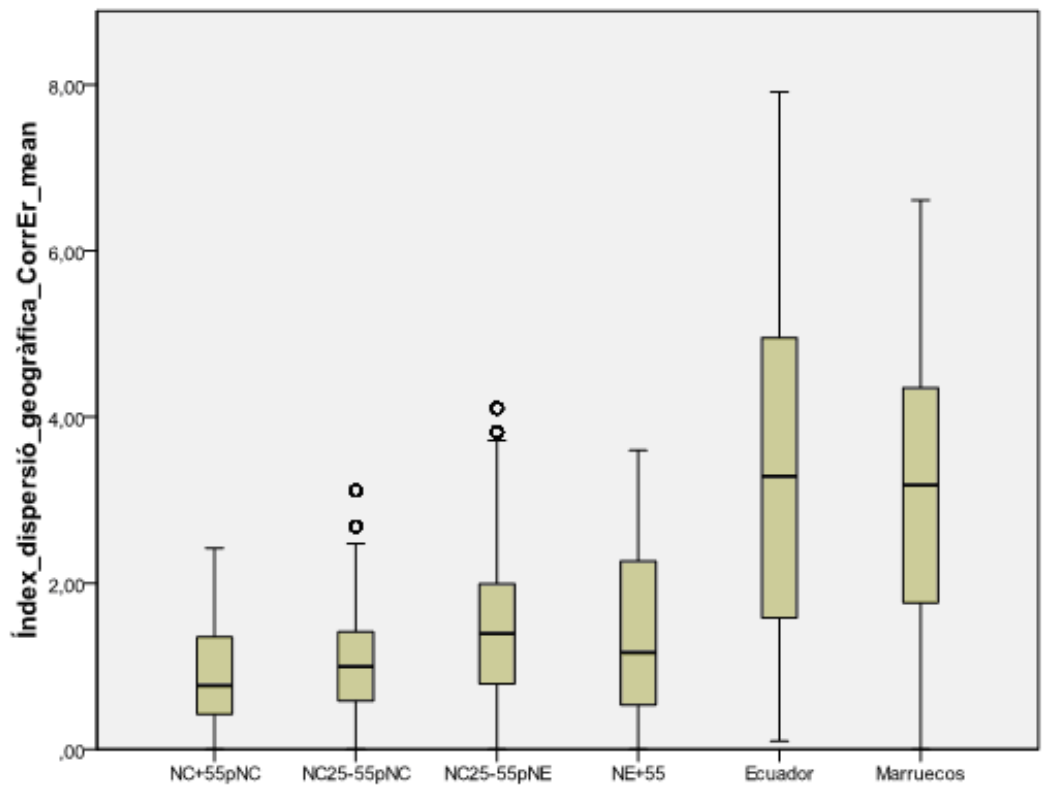

Figura 4. Diferencias en la distribución geográfica de la red personal en función del origen de los Ego según la el índice de dispersión Ego-Alter.

Pasemos ahora a examinar el tercer índice, el basado en un ranking de distancias.

\section{Ranking de distancias}


El tercer índice se muestra en la Figura 5. Las categorías para distribuir los Alteri son (el mismo) "barrio de Ego", "Municipio de Ego", "Cataluña", "España" y "Extranjero". Podemos observar cómo los datos son consistentes entre sí aplicando los diferentes índices ("glocalización" de nuevo). Este último tiene la ventaja de etiquetar cada grupo de Alteri y así, por ejemplo, nos muestra la importancia del grupo de Alteri situados en el "Extranjero" (una categoría negativa, dicho sea de paso) en comparación con los Alteri situados en España y que obedece a la importancia de la población emigrante en Cataluña procedente de otros países (Marruecos y Ecuador en este caso). Como sabemos, los contactos familiares en el país de origen se mantienen con el paso del tiempo y a pesar de la distancia, aunque ésta tiene un efecto de erosión en el largo plazo si no hay un mantenimiento de las relaciones a causa de los viajes, las visitas o los contactos ocasionales. Es lo que podríamos Ilamar un efecto de "gravedad lenta" que tiende a hacer coincidir contactos activos y proximidad geográfica en el largo plazo a menos que sea contrarestada por otros factores como pueden ser la movilidad laboral, los estudios superiores $\mathrm{u}$ otros factores.

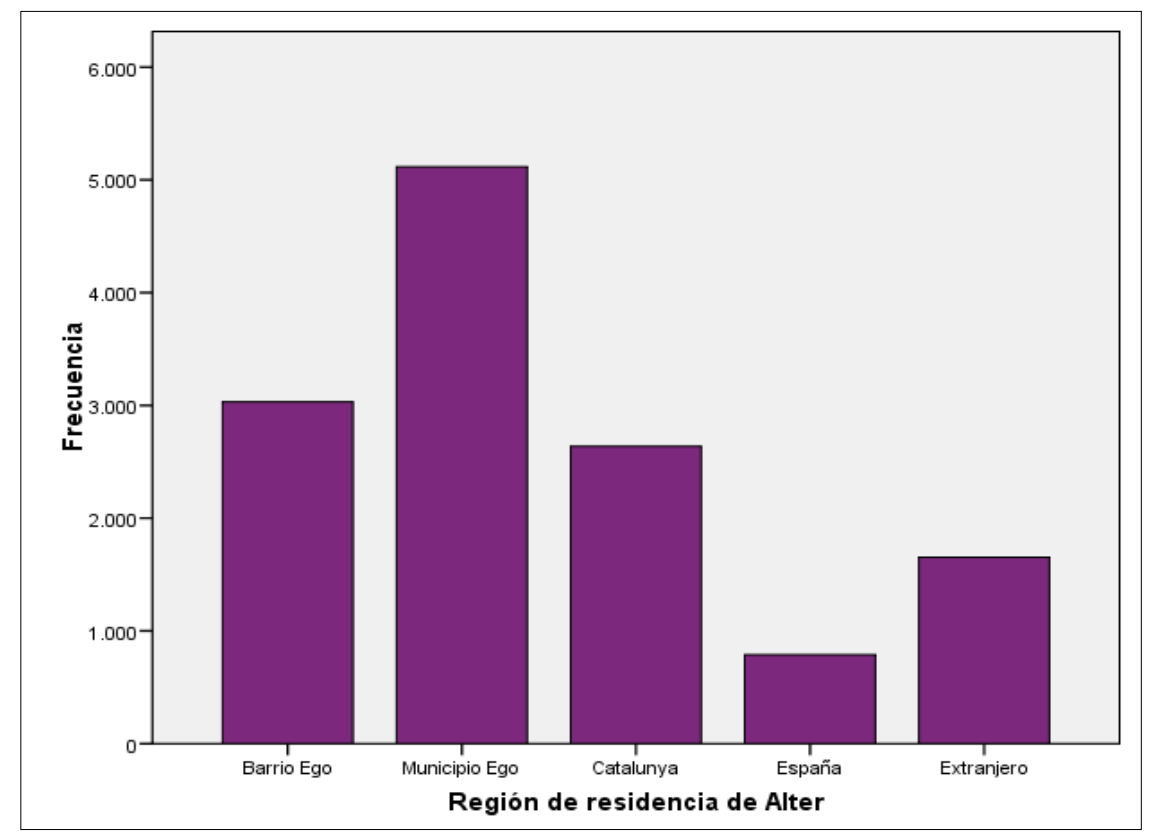

Figura 5. Frecuencia de la distribución de la variable "Región de residencia de Alter".

Si observamos la Figura 6, vemos de nuevo como los autóctonos tienen unas redes compuestas mayoritariamente por contactos locales, mientras que las redes de los inmigrantes se ubican repartidas entre lo local y lo transnacional, manteniendo una buena parte de los contactos activos en el extranjero. 


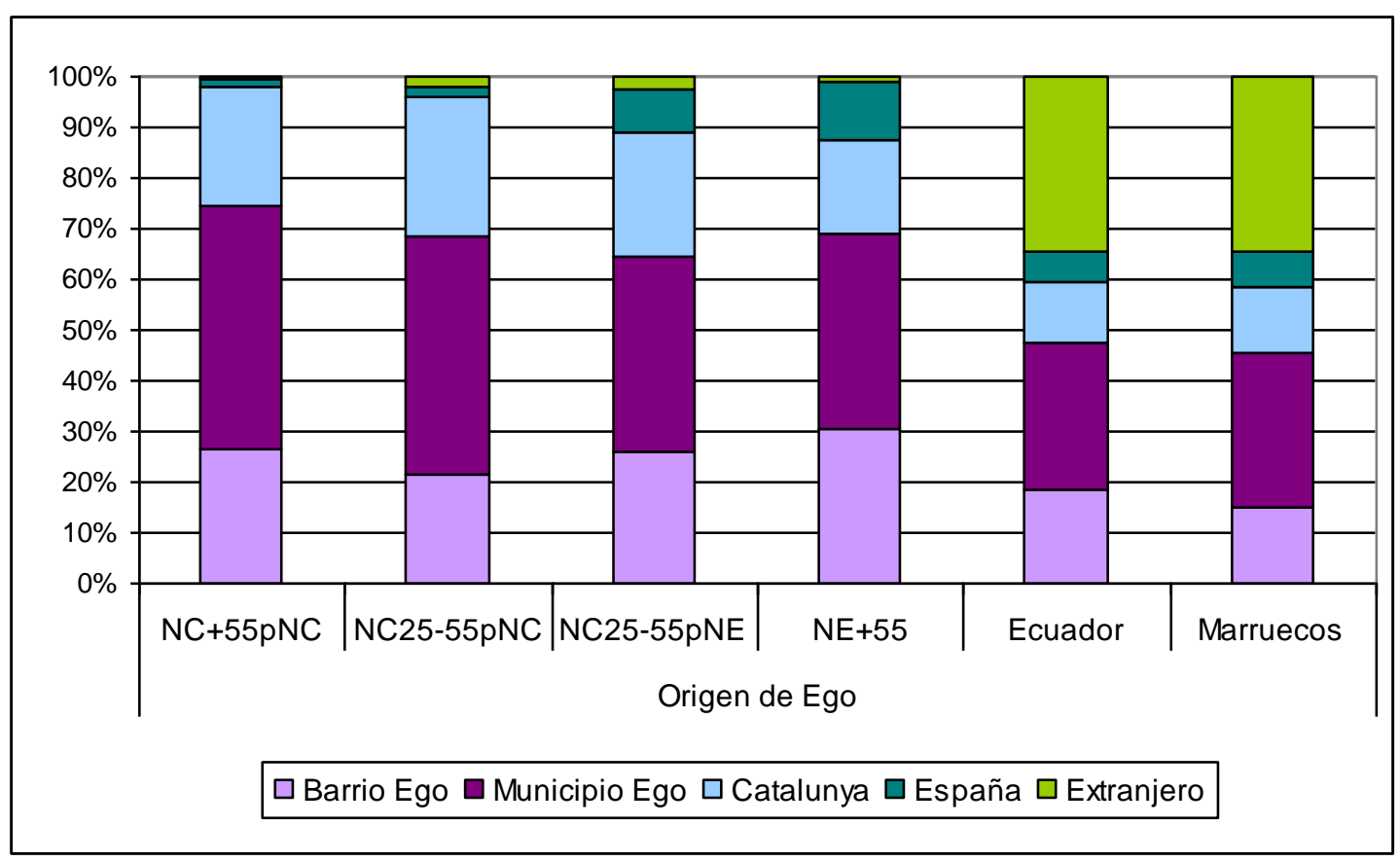

Figura 6. Diferencias en la distribución geográfica de la red personal en función del origen de los Ego según la región de residencia de los Alteri.

Así pues los tres índices son consistentes entre sí e indican la existencia de dos grandes realidades diferenciadas en lo que respecta al comportamiento en la distribución geográfica de las redes personales: la de la población nacional o autóctona (nacidos en Catalunya o en el resto de España) y la de la población extranjera.

Con el fin de comprobar si los índices de distribución geográfica son capaces de expresar algún fenómeno o realidad social más allá de caracterizar los grupos de origen, consideramos interesante la aplicación del índice separadamente en estos dos colectivos.

\section{Aplicación de las medidas de dispersión geográfica al estudio del apoyo social}

El concepto de apoyo social que utilizamos aquí se refiere a los recursos afectivos e instrumentales que se obtienen en la interacción social (Jariego, Martínez y García, 1999), tales como información, consejos, recursos materiales u otros tipos de ayuda. Así, entendemos por "red de apoyo social" el subconjunto de la red personal del individuo que proporciona información, ayuda instrumental y apoyo afectivo (Maya, 2006).

Las variables sobre apoyo que emplearemos a continuación informan del recuento total de Alteri en cada red $(n=30)$ de quienes se ha recibido o a quienes se ha 
ofrecido apoyo en cada ámbito. Hay que tener en cuenta que las variables hacen referencia a toda la extensión temporal de la relación, no a un periodo temporal determinado.

En la Figura 7 se observa la distribución de los Alteri en las categorías de los rankings de distancias o regiones geográficas, diferenciadas según el origen de los Egos con una línea oscura. Esta distribución geográfica de las redes nos sirve de referencia al analizar de dónde se recibe el apoyo. Observamos cómo la distribución del apoyo es muy parecida a la distribución de los Alteri, lo cual puede señalar cierta independencia del intercambio de apoyo respecto la distancia y ubicación de la relación. Como muestra la Tabla 1 , la localización de la red de apoyo se distribuye de forma muy diferente entre la población autóctona y la extranjera (X2 sig $=0,000, V$ Cramer $=0,486)$. La red de apoyo de la población nacional se ubica mayoritariamente en el ámbito local (casi un $70 \%$ de los Alteri que intercambian apoyo con ego se encuentran en el municipio de ego - incluyendo los del mismo barrio-), mientras que la subred de apoyo de la población inmigrada se mantiene en una buena parte (el $36,2 \%$ ) en el extranjero.

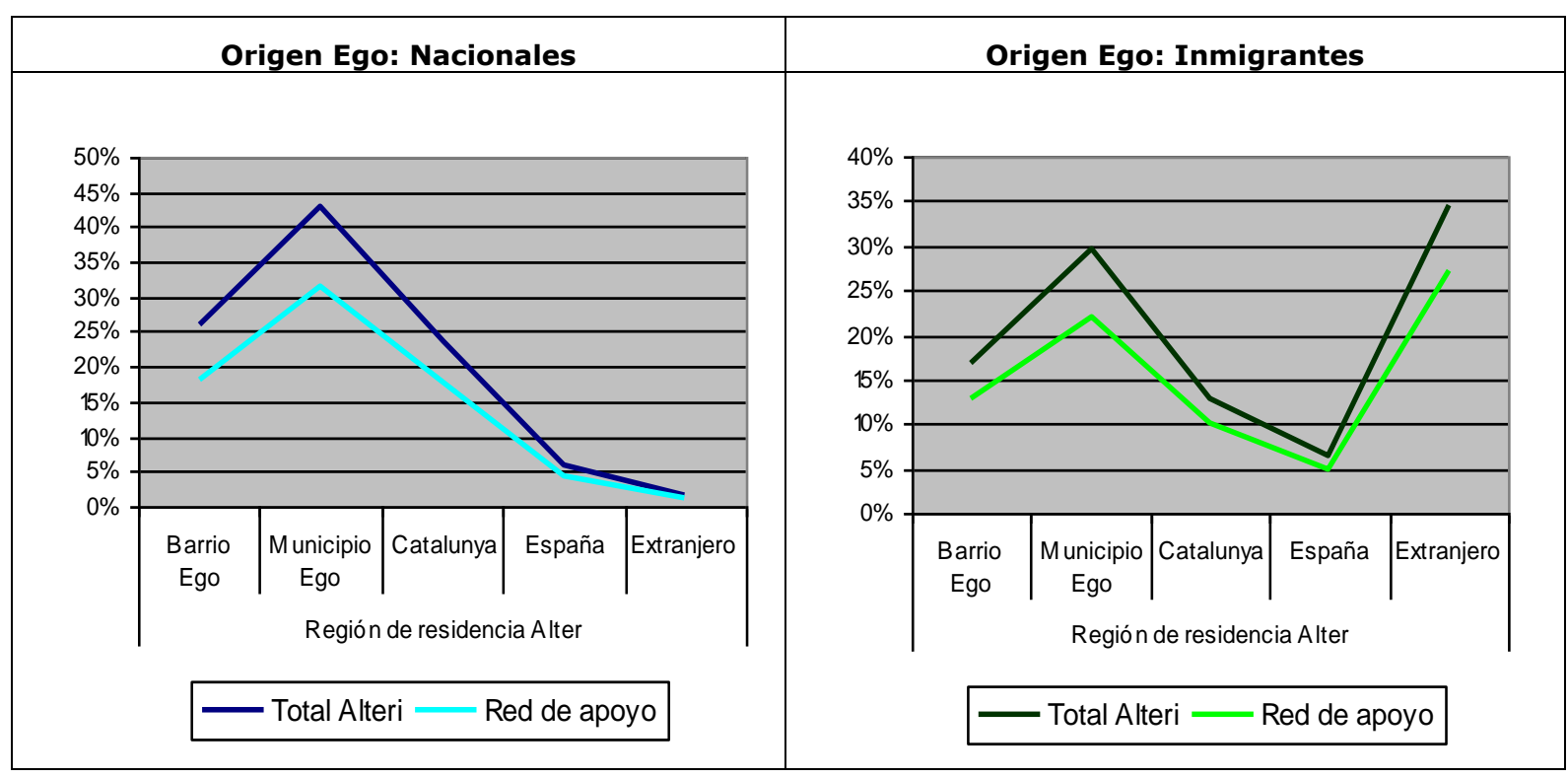

Figura 7. Distribución geográfica de la red de Ego teniendo en cuenta la totalidad de los Alteri y únicamente la subred de apoyo, para los nacionales y los extranjeros separadamente. 


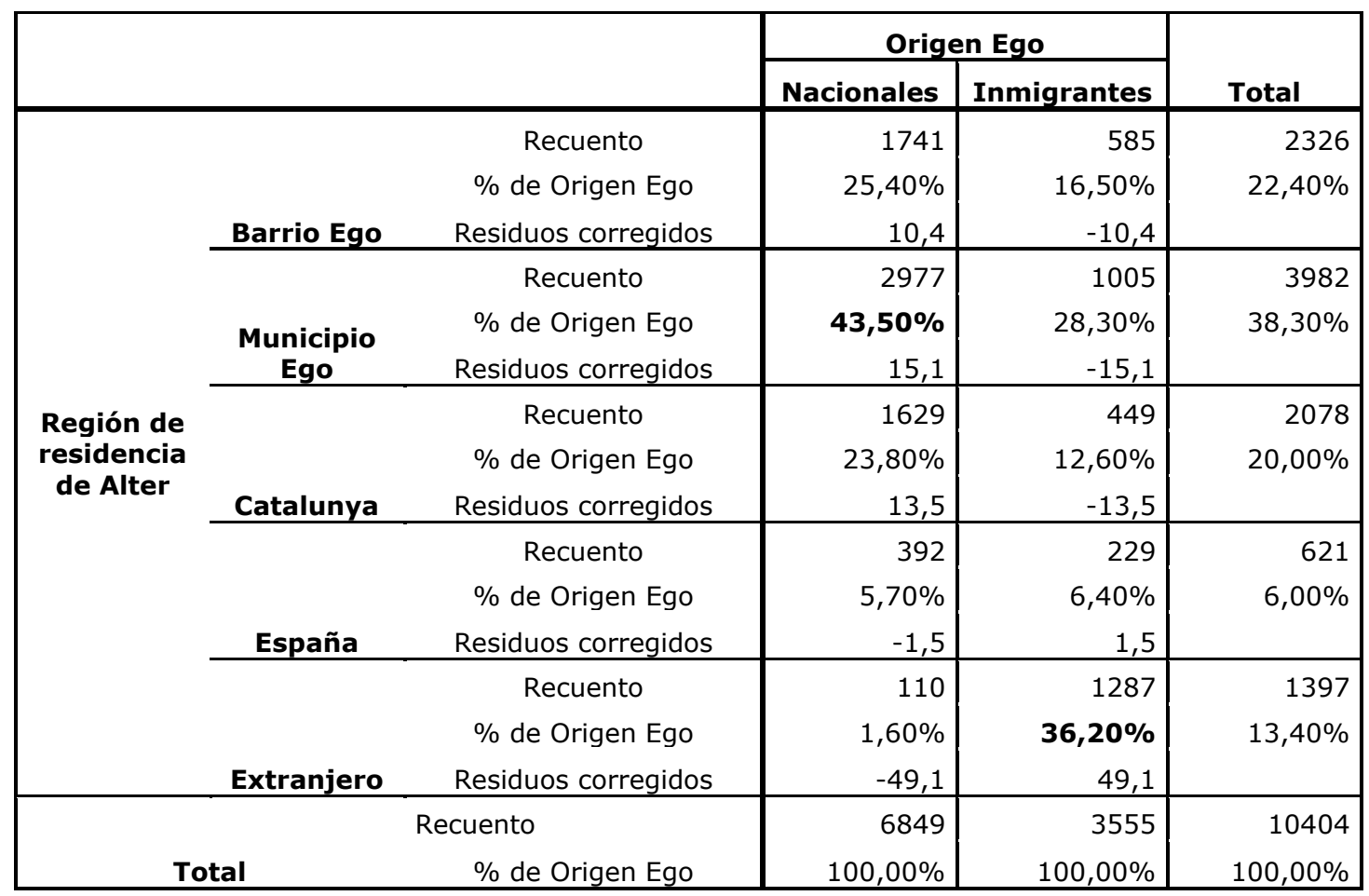

Tabla 1. Distribución espacial de la red de apoyo según el origen de los Egos.

Sin embargo, como muestra la Figura 8, la diferenciación de los distintos tipos de apoyo muestra diferencias significativas según la ubicación de los Alteri y el origen de Ego (X2 Sig.=0,000). Así, observamos cómo entre la población autóctona, el tipo de apoyo que se intercambia con una mayor proporción de Alteri es el relacionado con problemas personales y otros, seguido de las cuestiones de salud y trabajo. Para estos tipos de apoyo es destacable la importancia de los Alteri residentes en la ciudad, mientras que únicamente el apoyo relacionado con la vivienda y con los servicios sociales y administrativos se intercambia más con Alteri que residen en el mismo barrio de Ego que con los que residen en el resto del municipio.

La Figura 8 muestra el mantenimiento de redes de apoyo transnacionales en el caso de la población inmigrada a Catalunya. En este sentido es especialmente relevante el apoyo expresivo que se intercambia con Alteri en el extranjero. 


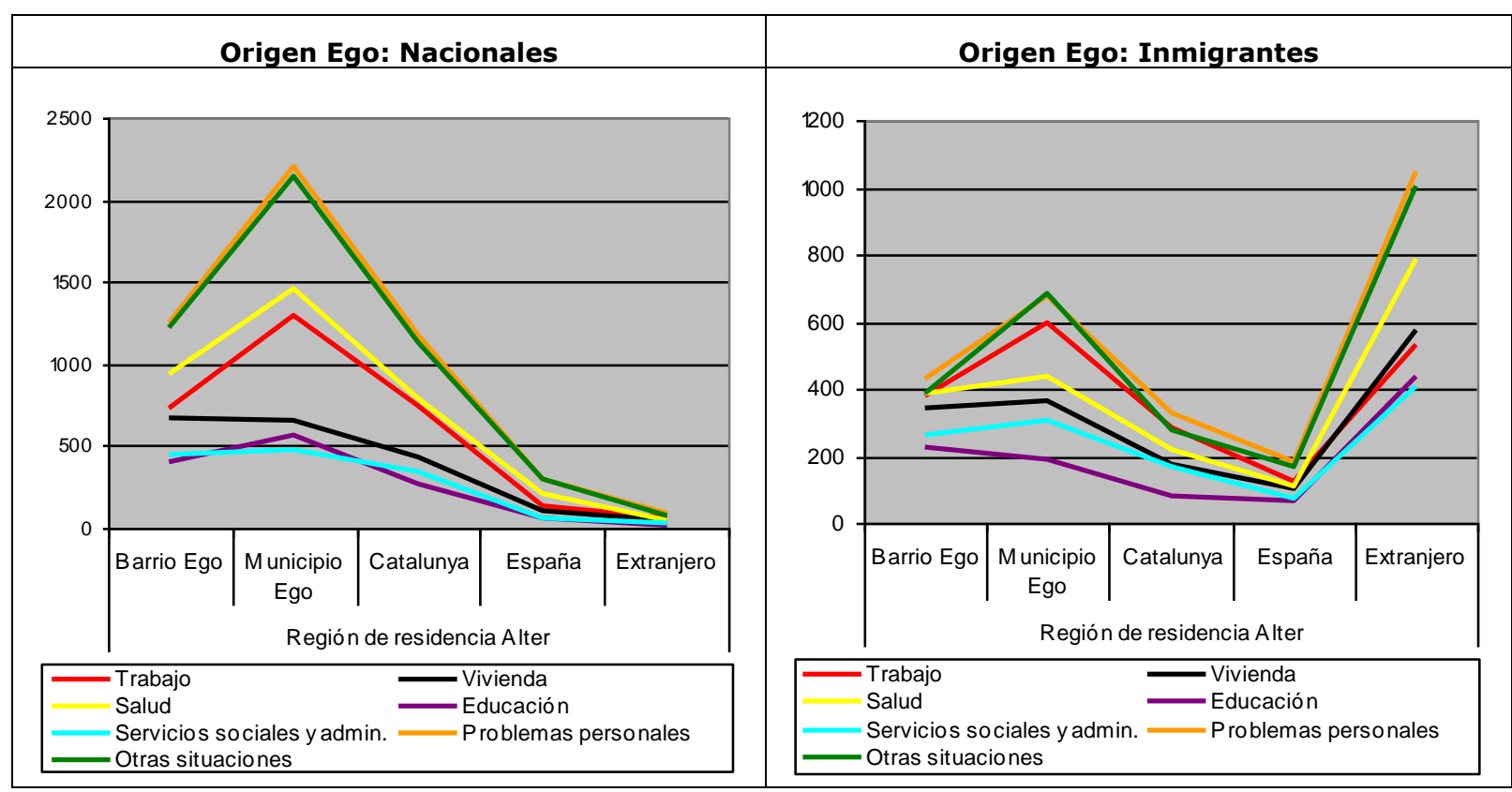

Figura 8. Distribución del apoyo Según la región de residencia de alter.

Una mirada más atenta sobre la proporción de Alteri con los que se intercambia apoyo social en cada región y según el tipo de apoyo nos enseña cómo, sorprendentemente, entre la población nacional la proporción de Alteri que residen en el extranjero con los que se mantiene una relación de intercambio de apoyo es muy elevada $(82,4 \%)$, incluso mayor que en las otras regiones. Este fenómeno se debe a que los Alteri que viven en el extranjero son contactos "fuertes", en muchos casos familiares, con los que, a diferencia de lo que se observa en otros países como Holanda (Mulder et.al., 2009), la distancia poco impacta sobre el intercambio de apoyo.

La Figura 9 muestra, para los extranjeros, como los Alteri con los que se intercambia una proporción de apoyo mayor son los que residen en el extranjero, con la excepción del apoyo relacionado con temas de trabajo y, en menor medida, de vivienda y servicios sociales y administrativos. También se observa cómo aquellos Alteri que residen en el mismo barrio de Ego en general intercambian apoyo con éste en una proporción mayor que con los que residen en el resto del municipio. Finalmente, una comparativa entre los autóctonos y los inmigrantes muestra cómo los inmigrantes intercambian una proporción bastante mayor de apoyo (entre un 15 y un $20 \%$ más) en lo que atañe a la obtención de empleo o información útil en temas de trabajo, vivienda y servicios sociales y administrativos, con los Alteri que residen en todas las regiones del país de destino. 


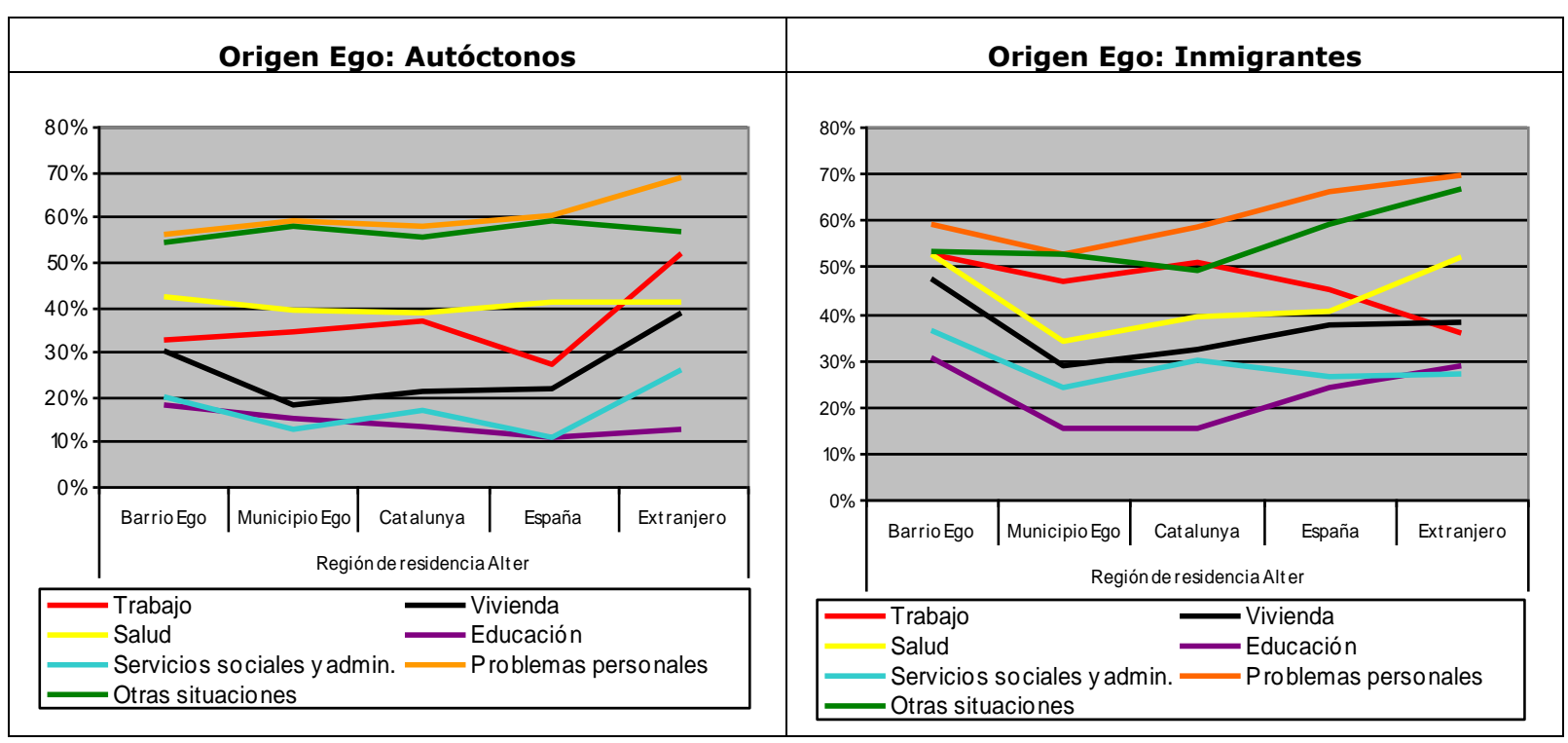

Figura 9. Porcentaje de los Alteri en cada región que han intercambiado apoyo con ego según el ámbito en que se circunscribe el apoyo.

Otra observación interesante es que, entre los inmigrantes, el apoyo que se intercambia con los Alteri que residen en su mismo barrio proviene de Alteri también inmigrantes, mientras que en el resto del municipio pesa un poco más el intercambio de apoyos con personas autóctonas, lo que puede ser una expresión de la segregación residencial (Martori y Hoberg, 2008).

Finalmente, haciendo un análisis de la evolución de la distribución geográfica de la subred de apoyo según el tiempo de residencia de la población inmigrante en Catalunya, la comparativa entre aquellos inmigrantes recién llegados respecto aquellos que llevan diez años o más en Catalunya nos muestra que hay cierta evolución en la distribución geográfica de la red de apoyo a medida que pasa el tiempo. Se observa cómo, en una primera etapa del proceso migratorio, se dispone de más Alteri en el extranjero que en el país de destino, mientras que a medida que pasa el tiempo la proporción se invierte, incrementando especialmente los contactos con personas del municipio. 


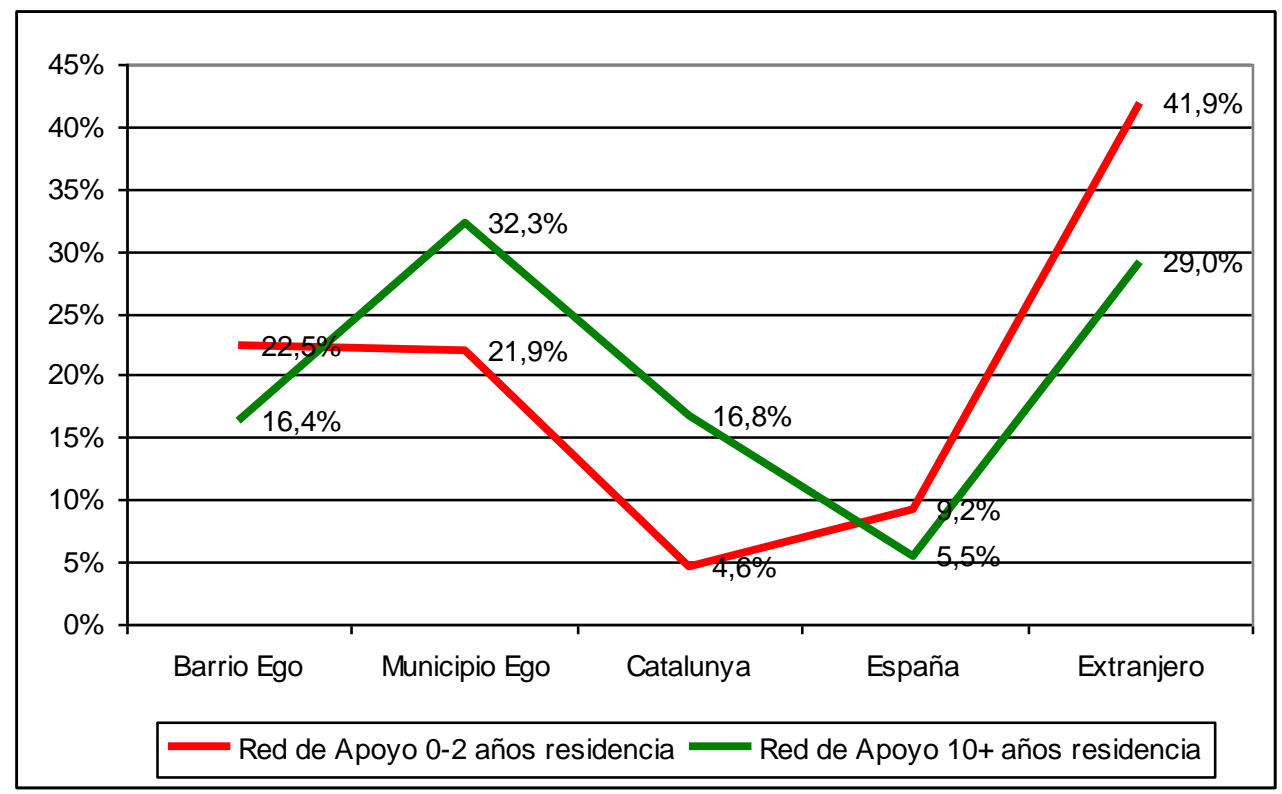

Figura 10. Distribución geográfica de la subred de apoyo en función del lugar de residencia de los Alteri según el tiempo de residencia en Catalunya.

La aplicación de los índices de dispersión geográfica ego-alter y alter-alter también muestran una tendencia a la reducción de la dispersión espacial de las redes a medida que se incrementa el tiempo de residencia en la sociedad de acogida. Así, entre aquellos extranjeros que han estado residiendo en el país durante menos de tres años, su índice es de 3,75; mientras que los que llevan más de 10 tienen un índice de 2,9. (Sig = 0.10). En cambio, la evolución de la dispersión geográfica alter-alter es mucho menor, no observamos diferencias significativas.

\section{Conclusiones}

Añadir la distancia geográfica al estudio de las relaciones sociales nos proporciona nuevas perspectivas y nos hace plantear nuevas preguntas. En general podemos decir que la dispersión geográfica de las redes personales captura no solamente el momento vital de Ego, sus decisiones individuales, las limitaciones o facilidades institucionales para su movimiento, sino también la dinámica de su círculo social. En el caso de las relaciones familiares, por ejemplo, resistiendo el paso del tiempo, en otros, sustituyendo personas, quizás con las mismas características (Lubbers et al. 2010).

Por lo que a los índices se refiere, cabe señalar que el índice basado en el ranking de distancias es muy informativo y permite caracterizar las relaciones sociales de los diferentes colectivos de forma rápida y precisa. En los otros dos casos, el índice "completo" y el índice Ego-Alter, hemos encontrado una alta correlación entre 
ambos al aplicarlo a los datos de población catalana (Correlación de Pearson 0,836; Sig. = 0.000). ¿Qué significan estos últimos dos índices? ¿Cómo interpretarlos? En el caso del índice Ego-Alter ya hemos avanzado que operacionaliza el concepto de "glocalización": desde la perspectiva de Ego, suma de forma lineal todas las distancias. El hecho que los resultados sean tan parecidos cuando se incluyen las distancias Alter-Alter se debe a que los dos casos de emigrantes estudiados tienen una distribución diádica, país de origen-país de destino (España y sus países próximos en este caso), con una baja proporción de Alteri distribuidos en otros países ( $11,0 \%$ de los contactos de los inmigrantes; $5,5 \%$ del total de la muestra). La medición de otros colectivos, con una distribución de los contactos activos más dispersa (diáspora) arrojaría diferencias mayores entre ambos índices. Dicho de otro modo: mientras que el índice Ego-Alter operacionaliza el concepto lineal de "glocalización", el índice de dispersión completo operacionaliza el concepto de "campo transnacional" propuesto por la teoría del transnacionalismo, a saber, un espacio de relaciones sociales más allá de las fronteras estatales que tiene una dinámica propia e influencias en varias direcciones. Cuanto más amplio sea este campo transnacional desde un punto de vista espacial (las distancias Alter-Alter), más diferencias habrán entre los dos índices.

Somos conscientes de las limitaciones del ejercicio que acabamos de presentar. En primer lugar es necesaria una simulación del comportamiento de los dos índices de dispersión para observar los patrones de comportamiento e identificar las condiciones a partir de los cuales es mejor utilizar uno u otro índice. Ya hemos apuntado que cuando las relaciones transnacionales son mayoritariamente diádicas (entre dos países), ambos índices son intercambiables.

En segundo lugar es necesario observar el comportamiento de los índices cuando en lugar de distancia geográfica se computa el coste de desplazamiento y ver en qué condiciones la distancia por sí sola es informativa y en qué condiciones no. Por último señalar que seguramente para que la distancia nos ayude a interpretar de manera diferente la realidad estudiada habrá que hacerlo de forma combinada con el tiempo, es decir, un análisis dinámico y espacial a la vez (Yoo et al. 2008) de las relaciones sociales.

\section{Bibliografía}

Altha J. Cravey, Sarah A. Washburn, Wilbert M. Gesler, Thomas A. Arcury, Anne H. Skelly (2001). "Developing socio-spatial knowledge networks: a qualitative methodology for chronic disease prevention", Social Science \& Medicine, Volume 52, (12), 1763-1775. 
Axhausen, K.W. (2008). "Social networks, mobility biographies, and travel: Survey challenges". Environment and Planning B-Planning \& Design, 35(6), 981-996.

Baybeck, Brady y Robert Huckfeldt (2002). "Urban contexts, spatially dispersed networks, and the diffusion of political information", Political Geography 21, 195220.

Brandes, U., Kenis, P., y J. Raab (2006). "Explanation Through Network Visualization", Methodology 2 (1), 16-23.

Carrasco, J. A., Hogan, B., Wellman, B., y E. J. Miller (2008). "Agency in Social Activity Interactions: The Role of Social Networks in Time and Space". Tijdschrift voor economische en sociale geografie, 99 (5), 562 - 583.

Chen, Y. D., Tseng, C. J., King, C. C., Wu, T. S. J., y Chen, H. C. (2007). "Incorporating geographical contacts into social network analysis for contact tracing in epidemiology: A study on Taiwan SARS data". Intelligence and Security Informatics: Biosurveillance, Proceedings, 4506, 23-36.

Clark, William A. V. y Suzanne Davies Withers (2009). "Fertility, Mobility and Labour-Force Participation: A Study of Synchronicity", Popul. Space Place 15, 305321.

Frändberg, L. (2008). "Paths in transnational time-space: representing mobility biographies of young Swedes". Geogr. Ann. B 90 (1), 1-28.

Glick Schiller, N., L. Basch y C. Szanton Blanc (1992) 'Transnationalism: a new analytic framework for understanding migration', en N. Glick Schiller, L. Basch y C. Szanton Blanc (eds), Towards a transnational perspective on migration: race, class, ethnicity and nationalism reconsidered, New York: New York Academy of Science, 1-24.

Glückler, J. (2007). "Economic geography and the evolution of Networks". Journal of Economic Geography, 7, 619-634.

Grossetti, M. (2006). "La ville dans l'espace des réseaux sociaux". En M. Bonnet y P. Aubertel (Eds.), La ville aux limites de la mobilité. Paris: Presses Universitaires de France, 83-90.

Grossetti, M. (2007). "Are French networks different?" Social Networks, 29 (3), 391-404.

Hampton, K., y B. Wellman (2002). "The Not So Global Village of Netville." En Wellman, B. Y Haythornthwaite, C (Eds.) The Internet in Everyday Life. Oxford: Blackwell, pp. 345-371.

Kleinberg, J. (2008). "The convergence of social and technological Networks". Commun. ACM, 51(11). Pp. 66-72.

Lambiotte, R., Blondel, V. D., De Kerchove, C., Huens, E., Prieur, C., Smoreda, Z., y P. Van Dooren (2008). Geographical dispersal of mobile communication network. arXiv:0802.2178v2 [physics.soc-ph].

Larsen, J., Urry, J., y Axhausen, K., (2006). Mobilities, Networks, Geographies. Ashgate: Aldershot. 
Levitt, P. y N. Glick Schiller (2004). "Conceptualizing Simultaneity: A Transnational Social Field Perspective on Society". International Migration Review, 38, (3), Conceptual and Methodological Developments in the Study of International Migration (Fall), pp. 1002-1039.

Liben-Nowell, D., Novak, J., Kumar, R., Raghavan, P., y Tomkins, A. (2005). "Geographic routing in social networks", Proc.Natl.Acad.Sci.U.S.A., 10, (33) Pp. 11623-11628.

Licoppe, C., Diminescu, D., Smoreda, Z., y Ziemlicki,C. (2008). "Using mobile phone geolocalisation for 'socio-geographical' analysis of co-ordination, urban mobilities, and social integration patterns. Tijdschrift Voor Economische" En Sociale Geografie, 99 (5), Pp. 584-601.

Lin, Nan; Ensel, Walter; y Vaughn, John. (1981). "Structural Factors in Occupational Status Attainment". American Sociological Review, Vol. 46 (4) pp. 393-405

Lozares, C.; J. M. Verd, P. López-Roldán, J. Martí y J. L. Molina (2011). "Cohesión, Vinculación e Integración sociales como formas de Capital social", REDES-Revista hispana para el análisis de redes sociales, vol. 20 (1) (http://revistaredes.rediris.es), enviado.

Lubbers M. J., J. L. Molina, J. Lerner, U. Brandes, C. McCarty y J. Ávila (2010). "Longitudinal analysis of personal networks. The case of Argentinean migrants in Spain". Social Networks, 31 (5).

Merton, Robert K. (1968). Social Theory and Social Structure. New York: The Free Press.

McCarty, Chris. (2002). "Structure in Personal Networks", JoSS. <http://www.cmu.edu/joss/content/articles/volume3/McCarty.html> [Consulta: 206-08].

Molina, José Luis; Ruiz, Alejandro A. y Laura Teves (2005). "Localizando geográficamente las redes personales", REDES-Revista hispana para el análisis de redes sociales, Vol.8, (5), <http://revista-redes.rediris.es/html-vol8/vol8_5.htm>.

Molina, JL, McCarty, Christopher y Eric Lavigne (2010). Utility for calculating the geographical dispersion index of personal networks collected with EgoNet. Grant: MICINN CSO2009-07057 - Perfiles del Empresariado Étnico en España (ITINERE).

Molina, J. L., I. Maya-Jariego y C. McCarty, (en prensa). "Giving Meaning to Social Networks: Methodology for Conducting and Analyzing Interviews based on Personal Network Visualizations". En B. Hollstein y S. Dominguez (eds.), Mixing methods in social network research, Cambridge University Press.

Mulder, Clara H. y Marieke J. van der Meer (2009). "Geographical Distances and Support from Family Members", Popul. Space Place 15, 381-399.

Niemegeers, I.E. y Heemstra de Groot (2002). "From Personal Area Networks to Personal Networks: A User Oriented Approach", Wireless Personal Communications 26, pp. 175-186.

- (2003). "Research Issues in Ad-Hoc Distributed Personal Networking", Wireless Personal Communications 26: 149-167. 
Obbo, C. (1993). "HIV transmission through social and geographical networks in Uganda". Social Science \& Medicine, 36 (7), 949-955.

Onnela J.P., Saramäki J., Hyvönen J., Szabó G., Lazer D., Kaski K., y Kertész J.,Barabási A.L. (2007). "Structure and tie strengths in mobile communication Networks". Proc Natl Acad Sci, 104 (18), Pp. 7332-6.

Urry, J. (2010). "Mobile Sociology", The British Journal of Sociology, 347-366.

Vertovec, Steven (2004). "Migrant Transnationalism and Modes of Transformation", International Migration Review, Vol. 38, (3) Pp. 970-1001.

Viry, Gil; Kaufmann, Vincent y Widmer, y Eric D. (2009). "Social integration faced with commuting: More widespread and less dense support networks". En Ohnmacht, Timo; Maksim, Hanja y Bergman, Manfred Max (Eds.) (2009). Mobilities and Inequality. Surrey: Ashgate.

Wimmer, Andreas y Nina Glick Schiller (2002). "Methodological nationalism and beyond: nation- state building, migration and the social sciences", Global Networks, Volume 2, (4) Pp. 301-334.

Yoo, JS y Hwang, J.M. (2008). "A framework for discovering spatio-temporal cohesive networks", Advances in Knowledge Discovery and Data Mining, Proceedings, 5012, Pp. 1056-1061.

Zorlu, Aslan (2009). "Ethnic Differences in Spatial Mobility: The Impact of Family Ties", Popul. Space Place 15, Pp. 323-342. 